\title{
Developing a three component stabilizer system for producing astaxanthin nanodispersions.
}

\begin{abstract}
Astaxanthin nanodispersions were prepared using Polysorbate 20 (PS20), sodium caseinate (SC) and gum Arabic (GA), solely or in combinations, as stabilizer system, through a solvent-diffusion process. The interactions among these three surface active compounds, in the formation, physicochemical and stability characterization of produced nanodispersions were studied by applying a simplex centroid mixture design. Quadratic or special cubic regression models were fitted for variations of all studied responses as function of significant $(\mathrm{p}<0.05)$ interaction effects between stabilizer components' proportions, with acceptable determination coefficients $(>0.70)$. Multiple-response optimization predicted that by using $29 \%(\mathrm{w} / \mathrm{w})$ PS20, $6 \%(\mathrm{w} / \mathrm{w}) \mathrm{GA}$ and $65 \%(\mathrm{w} / \mathrm{w}) \mathrm{SC}$ as a three component stabilizer system, an astaxanthin nanodispersion could be produced with the most desirable physicochemical characteristics and highest physicochemical stability. At this optimum stabilizer components proportions, the corresponding predicted response values for mean particle size, polydispersity index (PDI) and total astaxanthin loss were predicted to be $114.6 \mathrm{~nm}, 0.261$ and $680 \mathrm{mg} / \mathrm{L}$, respectively. The optimum astaxanthin nanodispersions also showed $2.06 \%$ and $1.05 \%$ particle size growth at $25{ }^{\circ} \mathrm{C}$ and $5{ }^{\circ} \mathrm{C}, 4.56 \%$ and $1.29 \%$ PDI growth at $25{ }^{\circ} \mathrm{C}$ and $5{ }^{\circ} \mathrm{C}$, and $20 \%(\mathrm{w} / \mathrm{w})$ astaxanthin loss at $25{ }^{\circ} \mathrm{C}$ after 8 weeks of storage. The absence of significant $(\mathrm{p}>0.05)$ differences between the experimental and predicted values of the response variables confirmed the adequacy of the fitted models.
\end{abstract}

Keyword: Astaxanthin; Emulsification-diffusion; Nanodispersion; Gum arabic; Sodium caseinate; Polysorbate 20. 